Revista Internacional de Apoyo a la Inclusión, Logopedia, Sociedad y Multiculturalidad.

Volumen 6, Número 3, diciembre 2020, ISSN: 2387-0907. DOI: https://dx.doi.org/10.17561/riai.v6.n3.3

\title{
Identificación y Tratamiento de las Necesidades Educativas Especiales de los Educandos
}

\begin{abstract}
Identification and Treatment of Special Educational Needs in the children
Identifizierung und Behandlung von sonderpädagogischem Förderbedarf bei den Kindern Identification et traitement des besoins éducatifs spéciaux des enfants Identificação e Tratamento de Necessidades Educacionais Especiais nas crianças Identificazione e trattamento di bisogni educativi speciali nei bambini Выявление и лечение специальных образовательных потребностей у детей 子どもたちの特別な教育的ニーズの識別と治療 识别和治疗儿童的特殊教育需要。
\end{abstract}

Cristina Merino Rodríguez Facultad de Humanidades y Ciencias de la Educación Universidad de Jaén cmr00047@red.ujaen.es

Fecha recepción: 14/10/2020

Páginas 24-41

Fecha aceptación: 29/11/2020

\section{Resumen.}

En el presente trabajo, se pretende destacar la importancia de la inclusión educativa en el aula ordinaria, por parte de los educandos que presentan Necesidades Educativas Especiales. Así como, se quiere resaltar la importancia de dar pautas de actuación para facilitar una verdadera inclusión educativa, además de cuestionar de cierto modo la supuesta formación que hay actualmente sobre este tema, señalándose así, la significativa labor docente. Asimismo, la investigación descriptiva llevada a cabo, aporta información de gran valor, aplicable a la vida cotidiana, para que de una u otra forma pueda ayudar y facilitar a las familias, docentes, futuros docentes y a la sociedad en general, datos importantes y conocimientos de interés. Por último, es muy relevante mencionar el rol fundamental que toma el diagnóstico de las diferentes características y peculiaridades que presente el alumnado para su posterior tratamiento.

Palabras clave: necesidades educativas especiales; inclusión educativa; investigación; alumnado; familia

\begin{abstract}
.
In this end of degree dissertation, the underlining of the significance of the inclusion education in the ordinary classroom is expected, aimed at the children with Special Educational Needs. Furthermore, it is highlighted the importance of offering protocols of action in order to facilitate a real inclusion education, as well as, question, in some way, the supposed formation on this topic, noting the importance of teachers' work. Moreover, this descriptive investigation gives valuable information on the daily life and, doing so, it can help by giving important and interesting information and knowledge to teachers, future teachers, families and, definitely, society in general.
\end{abstract}


Finally, it is very important the position of the diagnosis of differents characteristics in the students for their treattment.

Keywords: special educational needs; inclusion education; investigation; students; family

\section{1.-Introducción.}

A modo introductorio, este trabajo se ve inmerso en una investigación de carácter mixto, descriptivo y correlacional, pero, antes de iniciar el proceso de indagación y la utilización de métodos y técnicas, se ha realizado una previa búsqueda de información y contenido propio de la literatura, es decir, de toda la documentación, artículos y documentos que hablan sobre la temática en cuestión, para elaborar así una revisión bibliográfica introductoria, como se puede ver en el marco teórico.

En un segundo lugar, se empieza la investigación propiamente dicha, que comienza en el capítulo II del marco empírico. Se secuencia los objetivos, las variables, las hipótesis, la población y muestra, la metodología y todos los aspectos que van a ser tratados y de interés en todo el proceso de investigación, para de esta manera, concretar y fijar expresamente la finalidad e intenciones que se pretendían a priori.

Para la ejecución y puesta en marcha de la investigación, se fijó el problema al que se quería dar solución, en torno al cual se centra la presente investigación "¿Ayuda la Identificación de Necesidades Educativas Especiales de los niños a la manera de incluirnos en las aulas ordinarias?

Y, una vez fijado y delimitado el problema, se crean las técnicas de investigación y de recogida de datos, los cuales son el cuestionario de escala Likert y las entrevistas, creadas haciendo una preselección de los ítems propios del cuestionario. Por otro lado, los métodos empleados para la elaboración de la investigación son, el método descriptivo y el método correlacional y las técnicas empleadas son, tanto cuantitativas (cuestionario) como cualitativas (entrevista), de ahí, la naturaleza mixta del estudio.

Finalmente, se lleva a cabo la interpretación y validación de los datos y respuestas obtenidas con la aplicación IBM SPSS Statistics 25, iniciando así el análisis descriptivo, calculando la media, la moda y la desviación estándar propias de cada pregunta y, por otra parte, el análisis correlacional, comparando y construyendo las correlaciones Rho de Spearman, detallando las correlaciones más significativas y teniendo en cuenta qué ítems y aspectos correlacionan más entre sí, buscando así la causa y el motivo de ello.

Para concluir, con esa investigación, mayormente se pretende ayudar a comprender y dar la importancia que se merece a la inclusión educativa de los discentes con Necesidades Educativas Especiales en el aula ordinaria, sin necesidad de tener que acudir a un colegio de educación especial, promoviendo de esta manera, la inclusión 
y el aprendizaje valorando las diferencias, peculiaridades y características de los demás.

\section{2.-Marco teórico.}

\section{1.-Análisis y Evolución de las "N.E.E".}

Los antecedentes del principio de las N.E.E "Necesidades Educativas Especiales" se encuentran según la UNESCO (1994), en la Declaración de Salamanca y el Marco de Acción, en el que se garantiza que las personas que presenten alguna discapacidad o necesidad educativa especial, deben formar parte del sistema educativo, facilitando el acceso a los sujetos con dichas necesidades, en la práctica común de educación y aulas ordinarias, sin dejarlos al margen.

Según la UNESCO la educación inclusiva representa una vía de análisis y transformación de los sistemas educativos, con la finalidad de dar respuesta a la desemejanza de estudiantes que se encuentran en las aulas. El término inclusión es definido por la Unesco como: "Una estrategia dinámica para responder de forma proactiva a la diversidad de los estudiantes y concebir las diferencias individuales no como problemas, sino como oportunidades para enriquecer el aprendizaje" (2008, p.11).

Como señala Porras (2010), se entiende el concepto de integración como el intento de normalizar la vida de los educandos que presentan necesidades especiales de ayuda educativa; mientras que el objetivo de una verdadera inclusión es el de presentar la educación como un derecho de todos los sujetos, tengan o no alguna discapacidad o dificultad de aprendizaje. (Citado en Olovarría, 2015, p.150).

Como bien menciona Chiner (2011), ha existido un cambio gigantesco entre el concepto "integración" y la visión actual que se tiene de la verdadera "inclusión". Se pensaba que la integración era el movimiento idóneo para llegar a tener una educación plena más allá de las diferencias que presentara cada niño, pero, realmente, la integración reconocía las diferentes discapacidades como un rasgo atípico 0 anormal de las personas, que de ningún modo ha logrado terminar con la exclusión. Por ello, surgiría el concepto de "inclusión", que hace que las personas sean valoradas por lo que son, dentro de los distintos contextos y no por presentar determinadas diferencias en comparación con el resto.

En definitiva, Chiner (2011) hace referencia a la palabra "inclusión" como aquella, sinónimo de derecho humano y justicia social, con equidad de posibilidades para todos los alumnos, implicando un cambio de actitudes y comportamientos en el entorno más próximo al educando y en la sociedad, en general.

Con respecto a la Comisión de Expertos, la definición de Necesidades Educativas Especiales se suele utilizar como equivalente a discapacidad, centrada en los niños que la padecen, ya que son incontables los casos que hay de referirse a los sujetos que muestran Necesidades Educativas Especiales vinculadas o no a discapacidad. 
Por lo que, sigue haciéndose referencia primordialmente a las condiciones personales del niño y no, a las situaciones sociales o educativas a las que está expuesto diariamente $(2004$, p.17).

\section{2.-Diversidad y Tipología de Necesidades Educativas Especiales.}

Mencionados los diferentes enfoques para interpretar las distintas Necesidades Educativas Especiales según la perspectiva de la Comisión de Expertos, se hace fundamental mencionar y hacer énfasis en los diferentes tipos de Necesidades Educativas Especiales que puede presentar el alumnado y que son necesarias tratar, dentro del aula ordinaria, mediante apoyo educativo, ya que son múltiples y muy variadas dentro de cada categoría.

Según las Instrucciones de la Dirección General de Participación y Equidad existen cuatro grandes grupos de alumnado que presentan necesidades específicas de apoyo educativo, dentro de los cuales, hay diferentes subgrupos (2017, p.151-152):

Alumnado con Necesidades Educativas Especiales.

Trastornos graves del desarrollo: Trastornos del desarrollo del lenguaje, retrasos evolutivos profundos o graves y trastornos del desarrollo psicomotor.

Discapacidad visual: Ceguera o baja visión.

Discapacidad intelectual: Discapacidad intelectual moderada, leve, grave 0 profunda.

Discapacidad auditiva: Sordera o hipoacusia.

Trastornos de comunicación: Afasias, trastornos del habla (disglosias o disfemias, disartrias) y trastornos específicos del lenguaje (expresivos, mixtos o semánticopragmático).

Discapacidad física: Lesiones de origen medular o cerebral, trastornos neuromusculares y lesiones del sistema osteoarticular.

Trastornos del Espectro Autista: Autismo, síndrome de Rett síndrome de Asperger, trastorno generalizado del desarrollo no especificado y trastorno desintegrativo infantil.

Trastornos graves de conducta: Trastorno disocial, trastorno de comportamiento perturbador no especificado y trastorno negativista desafiante.

Trastorno por déficit de atención con hiperactividad "TDAH": Predominio del déficit de atención, de la impulsividad - hiperactivad o tipo combinado.

\section{Otros trastornos mentales.}

\section{Enfermedades crónicas y raras.}


Alumnado con dificultades de aprendizaje.

Dificultad específica de aprendizaje: De la lectura - dislexia, en el aprendizaje de la escritura - disortografía, de la escritura - disgrafía y en el aprendizaje del cálculo discalculia.

Dificultad de aprendizaje por capacidad intelectual límite.

Dificultad de aprendizaje por retraso en el lenguaje.

Dificultad de aprendizaje derivadas de trastornos por déficit de atención con 0 sin hiperactividad.

Alumnado con altas capacidades intelectuales.

\section{Talento Complejo.}

\section{Sobredotación Intelectual.}

\section{Talento Simple.}

\section{Alumnado que necesita acciones de carácter compensatorio.}

Mencionado todo lo anterior, dichas Instrucciones del 8 de Marzo de 2017 definen a los discentes que presentan Necesidades Educativas Especiales como aquellos que necesitan, toda su escolarización o únicamente un periodo de ella, una atención específica en su educación, como consecuencia de sus trastornos graves de conducta o desarrollo, discapacidad o TDAH. (p. 153).

\section{3.-¿Escasez y Falta de Información?}

La gestión de la información y conocimiento acerca de los educandos que presentan Necesidades Educativas Especiales debe ser adecuada, para así, efectuar las adaptaciones curriculares pertinentes, valorando el aprendizaje y el proceso formativo individualizado. Para ello, se ha de tener en cuenta la predisposición favorable del profesorado para la inclusión escolar, para desenvolver de este modo, las adaptaciones de tipo didáctico, organizativo y evaluativo, para así conseguir un proceso de enseñanza-aprendizaje inclusivo y significativo. (Wenger, 2002; Molina, 2009. Citado en Rodríguez y Ortiz, 2017).

A la pregunta "¿existe una verdadera inclusión educativa?", Alonso y Aguilella (2008) dan respuesta. Estos autores mencionan que la inclusión educativa requiere un trabajo y esfuerzo diarios, no solo por parte de la escuela, sino de las familias y de toda la sociedad en su totalidad, ya que los cambios en el sistema educativo tienen lugar de manera lenta y para ello, es necesario el desarrollo de ciertas estrategias y procedimientos para llegar a satisfacer plenamente la atención de los infantes con Necesidades Educativas Especiales.

Además, cabe señalar que no es suficiente que el alumnado aprenda determinadas competencias diferentes para compartir el currículum ordinario de la educación 
general, sino que la inclusión se puede ver favorecida modificando ciertos aspectos y características ambientales de los contextos en los que se desenvuelve el sujeto en cuestión. De manera que así aparece el concepto de inclusión, haciéndose fundamental cambiar los estereotipos y prejuicios que la sociedad tiene y así, mejorando la cualificación y preparación profesional, que den lugar a una mejora significativa en la programación educativa inclusiva. (Alonso y Aguilella, 2008).

Según Alonso y Aguilella (2008), los familiares de los educandos que presentan algún tipo de Necesidad Educativa Especial "N.E.E" consideran que hoy en día hay un desconocimiento generalizado acerca de lo que es y supone una verdadera inclusión educativa. Además, se cree que la predisposición docente es fundamental para progresar en la inclusión y hacen alusión a que la formación e implicación docente no es la suficiente para poder abordar este tema de tal envergadura. Consecuentemente, las familias demandan medios humanos y materiales para poder atender al alumnado correctamente de manera individualizada. Así como, las familias solicitan más información y pautas para poder ayudar a sus pequeños, para de este modo, existir una relación bidireccional entre la familia y la escuela, que en algunas ocasiones resulta un problema por ambas partes.

Por ello, Alonso y Aguilella (2008) proponen que lo idóneo de que la escolarización de los niños sea en un centro ordinario y no en un colegio especializado es, que se le brinda un abanico de oportunidades que hace que desarrollen al máximo sus posibilidades, y, por lo tanto que, la inclusión sea siempre algo implícito dentro de su educación, por lo que una falta de formación o concienciación sobre la atención y respeto de la diversidad, supone un problema directo para los familiares y, consecuentemente, un conflicto con sus hijos e hijas para poder solventar las distintas dificultades que puedan surgir y surjan.

Por esta razón, las familias reclaman más información y que se le dé la importancia que tiene las necesidades de sus niños para poder sentirse partícipes con la comunidad educativa y poder colaborar con el profesorado, para así provocar que la inclusión se efectúe como una práctica real en las aulas y que el profesorado se centre más en la inclusión social, la creación de amistades y no tanto en los contenidos académicos, de ahí que los familiares demanden pautas para poder trabajar con los niños desde casa, para así mejorar y conseguir una educación tanto individualizada como en equipo. (Alonso y Aguilella, 2008).

\section{3.-Marco Empírico.}

\section{1.-Problema.}

Según lo estudiado en el marco teórico con los diferentes autores mencionados y trabajados, se ha de aludir que el problema en torno al cual va a ir centrada la presente investigación será, si la identificación de las diversas y múltiples Necesidades Educativas Especiales de los niños, ayuda y facilita la manera de incluirlos en las aulas ordinarias. Es decir: 
Problema: "¿Ayuda la identificación de Necesidades Educativas Especiales de los niños a la manera de incluirlos en las aulas ordinarias?"

De tal modo que, para orientar esta investigación se van a tener en cuenta dos variables. Primeramente, la variable independiente $(\mathrm{VI})$, va a ser la variable que se observe para ver su relación con la variable dependiente. $Y$, en segundo lugar, la variable dependiente (VD), será la variable que se mida en función de los valores y cambios que sufra la variable independiente. En otras palabras, se quiere conocer cómo la forma de tratar e integrar a los educandos en las aulas ordinarias, se ve afectada por las diferentes características y tipología que presentan las Necesidades Educativas Especiales.

VI: Necesidades Educativas Especiales.

VD: Forma de tratar e integrar.

\section{2.-Objetivos.}

En función del problema planteado y de la previa información obtenida para el planteamiento de esta investigación, se enumeran seguidamente los objetivos que se pretenden conseguir.

\subsection{1.-Objetivo general y Objetivos Específicos.}

En primer lugar, se detalla el objetivo general y central de la investigación:

- Identificar las Necesidades Educativas Especiales que presentan los niños.

En cuento a los objetivos específicos, se enumeran concretamente cinco objetivos específicos, que, de manera más detallada, muestran la finalidad y el propósito de la puesta en práctica de esta investigación:

1.Mostrar cómo influye el ambiente a las diferentes dificultades de los niños.

2.Distinguir los numerosos trastornos del desarrollo que tienen lugar en el aula.

3.Explicar la forma de actuar ante las diferentes Necesidades Educativas Especiales.

4.Analizar cómo se lleva a cabo el apoyo a la inclusión en el aula ordinaria.

5.Contrastar la inclusión en la escuela e incrementar el conocimiento sobre las Necesidades Educativas Especiales para los miembros de la Comunidad Educativa.

De cada uno de los objetivos específicos propuestos, se ha extraído una línea de investigación, a partir de las cuales se ha llevado a cabo la previa búsqueda de información y documentación acerca de todo lo relacionado con la actual investigación. Se pueden comprobar en la siguiente tabla: 
Tabla 1.-Objetivos específicos y sus correspondientes líneas de investigación.

\section{OBJETIVOS ESPECÍFICOS LÍNEAS DE INVESTIGACIÓN}

1. Mostrar cómo influye el ambiente a las diferentes A. Ambiente dificultades de los niños

2. Distinguir los numerosos trastornos del desarrollo que tienen lugar en el aula

B. Trastornos del

3. Explicar la forma de actuar ante las diferentes Necesidades Educativas Especiales

4. Analizar cómo se lleva a cabo el apoyo a la inclusión en el D. Inclusión aula ordinária

5. Contrastar la inclusión en la escuela e incrementar el conocimiento sobre las Necesidades Educativas Especiales para los miembros de la Comunidad Educativa desarrollo

C. Acción

Fuente: elaboración propia.

\section{3.-Hipótesis.}

Una vez avanzada la investigación, en lo que respecta a las hipótesis, se han planteado dos, la primera contraria a la segunda, ya que lo que se pretende verificar y contrastar es si la identificación y detección de las Necesidades Educativas Especiales de los educandos, facilita o no, la inclusión educativa en el aula ordinaria. Son las que se muestran a continuación:

HO: La detección de Necesidades Educativas Especiales ayuda a la manera de incluir a los niños en el aula ordinaria.

H1: La detección de Necesidades Educativas Especiales NO ayuda a la manera de incluir a los niños en el aula ordinaria.

\section{4.-Población y muestra.}

En relación con la población y la muestra seleccionada para llevar a cabo la investigación. En un primer momento, se optó por la población $(N)$ residente en la provincia de Jaén, pero debido al amplio y gran número de individuos, se hizo una criba para hacer una selección mucho más característica y personalizada al interés del estudio. De tal manera que, se realizó un muestreo intencional, llevando a cabo una selección de participantes más representativos y típicos de la población a la que se quiere hacer referencia con esta investigación.

Consecuentemente, la muestra (n) y participantes que han colaborado en esta investigación en representación de la sociedad han sido 227 sujetos concretamente, 
de entre los cuales se encuentran futuros docentes del Grado de Educación Infantil y familias y profesorado de diferentes colegios y centros educativos, pertenecientes a la provincia de Jaén.

La muestra fue seleccionada puesto que son un grupo de personas que representan las características propias de la sociedad, en lo que respecta al ámbito educativo, social y familiar, por lo tanto, los participantes comparten la peculiaridad de estar inmersos en el sistema educativo actual, bien por ser docentes, futuros docentes 0 familiares de educandos, por lo tanto, presentan y demuestran las competencias necesarias en lo que concierne a la educación.

\section{5.-Metodología.}

Partiendo de la metodología empleada en esta búsqueda, se ha de decir que el presente estudio se ve inmerso en una investigación mixta, descriptiva y correlacional, puesto que las técnicas de investigación utilizadas son técnicas propias de una metodología tanto cuantitativa como cualitativa.

En lo que respecta a las técnicas de investigación cuantitativas, se ha usado el cuestionario como herramienta de observación, medición y análisis de variables. Y, en cuanto a las técnicas cualitativas, se ha empleado la entrevista, usándola con su guía más estructurada, para conocer de manera más amplia, personal y subjetiva el conocimiento que se tiene acerca del tema tratado.

El método aplicado para la puesta en marcha de la investigación ha sido un método descriptivo, explorando, asociando y comparando los grupos de datos. Así como, el método correlacional, mediante el cual se establecen las diversas relaciones entre variables, para conocer la influencia que tienen unas sobre otras.

\section{6.-Instrumentos.}

En lo que respecta a las herramientas empleadas para la recopilación y recogida de datos, se destaca la utilización de técnicas de autoinforme, es decir, aquellas en las que los sujetos muestran su opinión y parecer sobre las cuestiones planteadas. Estas técnicas han sido concretamente el cuestionario de escala Likert y la entrevista, ambos instrumentos fueron elegidos ya que son técnicas que permiten observar con claridad las respuestas y pensamientos de los participantes en la investigación, así como, las dos técnicas son de creación propia.

En primer lugar, en relación con el cuestionario de escala Likert, se ha de mencionar que fue confeccionado en un documento WORD, empleando la escala Likert para mostrar el grado de conformidad en las respuestas de los individuos, siendo esta "Muy de acuerdo (1)", "Desacuerdo (2)", "Indiferente (3)", "De acuerdo (4)" y "Muy de acuerdo (5)". En un primer momento, estos cuestionarios fueron entregados a la población mencionada con anterioridad, donde se iban a llevar a cabo la validación de los mismos, pero, debido a la alarmante situación provocada por el COVID-19, se realizó mediante Encuestas Google, haciéndolos llegar a los sujetos participantes del 
presente estudio. Por lo tanto, los datos y respuestas se validaron y recogieron en un documento EXCEL, respuesta por respuesta e ítem por ítem, para conseguir así su posterior análisis de resultados con la aplicación IBM SPSS Statistics 25.

En segunda instancia, la entrevista fue elaborada también en WORD y esta se hizo haciendo una selección de los ítems utilizados en el cuestionario. Las diferentes entrevistas se iban a hacer con las personas entrevistadas personalmente, de manera personal, pero a consecuencia de las circunstancias, se realizaron por llamada telefónica, en concreto, la entrevista la efectuaron a nueve personas y se validó haciendo las interpretaciones pertinentes de las respuestas aportadas por los participantes. Dichas interpretaciones se mostrarán en el apartado de análisis de resultados, junto con el análisis descriptivo y el análisis correlacional de los cuestionarios.

\subsection{1.-Ítems del Cuestionario de la Escala Likert.}

Haciendo referencia al cuestionario de escala Likert propiamente dicho, se ha de mencionar que, se confeccionó siguiendo las 5 líneas de investigación mencionadas anteriormente. De tal manera que, se elaboraron 5 ítems para cada una de las líneas, dando lugar así a un total de 25 ítems para la ejecución del cuestionario. Los ítems son los que se contemplan seguidamente:

Ítems:

\section{A. Ambiente.}

A1: El ambiente familiar y social en el que se desarrolla un determinado niño/a influye de manera directa en la educación del mismo.

A2: NO debe existir una relación bidireccional entre familia y escuela.

A3: Los padres y familiares de un niño/a con N.E.E (Necesidades Educativas Especiales) deben facilitar al centro la información necesaria para ayudarlo en su educación.

A4: Las habilidades sociales de los niños/as se ven claramente afectadas por su entorno más cercano.

A5: El contexto y circunstancias en las que se ven envueltos los niños/as condicionan de manera directa su comportamiento y habilidades sociales.

B. Trastornos del desarrollo.

B6: Soy consciente de los diferentes tipos de Necesidades Educativas Especiales que puede padecer un niño/a en un aula ordinaria.

B7: La detección de Necesidades Educativas Especiales de los niños/as facilita su inclusión en el aula ordinaria. 
B8: Sería capaz de identificar que un niño tiene algún tipo de trastorno 0 discapacidad con la información que poseo hoy en día.

B9: Antes de rellenar este cuestionario, NO sabía a qué hacía referencia las siglas N.E.E.

B10: Nunca llegaría a aceptar que mi hijo tuviese una determinada discapacidad.

C. Acción.

C11: Si me encuentro con un niño/a con una determinada discapacidad (visual, auditiva, intelectual...), sabría cómo reaccionar y tratarlo.

C12: Pienso que hoy en día los docentes tienen los medios y recursos necesarios para poder trabajar con niños/as con N.E.E.

C13: Conozco la forma de actuar ante cada uno de los diferentes tipos de Necesidades Educativas Especiales.

C14: Las acciones y actitudes con los niños con N.E.E necesitan un cambio para llegar a una verdadera inclusión educativa en el aula ordinaria.

C15: Se debería dar pautas de actuación y aceptación para llegar a una mayor concienciación sobre las Necesidades Educativas Especiales.

D. Inclusión.

D16: El comportamiento del profesorado influye en la inclusión de los niños/as con N.E.E en el aula y en el colegio.

D17: Hoy en día no se lleva a cabo una verdadera inclusión en el aula ordinaria.

D18: Tengo una amplia información acerca de si mi hijo/a asiste a un colegio inclusivo.

D19: El maestro/a de mi hijo/a acepta plenamente la inclusión en el aula.

D20: Yo como familiar de mi hijo/a, sobrino/a... transmito el valor y respeto hacia los niños/as con cualquier tipo de Necesidad Educativa Especial en su aula ordinaria.

E. Conocimiento e información.

E21: Creo que sería adecuado una mayor información y conocimiento acerca de todo lo relacionado con las N.E.E de los niños/as.

E22: Todos los colegios deberían promover charlas para adquirir conocimiento sobre lo ideal de una escuela inclusiva. 
E23: Me gustaría obtener más información sobre las distintas discapacidades 0 necesidades que puede poseer un niño/a y que pueden tener lugar dentro del aula ordinaria.

E24: La sociedad no es consciente de que un niño con una determinada discapacidad no es el problema, sino la escasez de información y concienciación.

E25: Actualmente existe una verdadera inclusión en la escuela ordinaria.

\subsection{2.-Ítems seleccionados para la Entrevista.}

De entre los 25 ítems nombrados, para la confección de la entrevista, se eligieron 10 ítems, precisamente 2 de cada línea de investigación, los considerados más significantes y de los que se podían extraer las respuestas individuales más sustanciosas y relevantes. Se escogieron los siguientes:

A1. ¿El ambiente familiar y social en el que se desarrolla un niño influye en su educación?

A5. ¿El contexto inmediato en el que crece y se educa un niño influye en su comportamiento y habilidades sociales?

B9. Antes de rellenar este cuestionario o esta entrevista, ¿sabía a qué hacían referencia las siglas N.E.E?

B10. ¿Hubieras aceptado que tu hijo/a tuviera algún tipo de discapacidad?

C12. ¿Crees que hoy en día los docentes tienen los medios y recursos necesarios para poder trabajar con niños/as con N.E.E?

C14. ¿Consideras que las acciones y actitudes que se llevan a cabo con los niños que presentan N.E.E necesitan un cambio para llegar a una verdadera inclusión educativa en el aula ordinaria?

D16. ¿El comportamiento del profesorado influye en la inclusión de los niños/as con N.E.E en el aula y en el colegio?

D20. Como familiar de mi hijo/a, sobrino/a... ¿transmites el valor y respeto hacia los niños/as con cualquier tipo de Necesidad Educativa Especial en su aula ordinaria?

E21. ¿Es necesario una mayor información y conocimiento acerca de todo lo relacionado con las N.E.E de los niños/as?

E24. ¿Consideras que la sociedad no es consciente de que un niño con una determinada discapacidad no es el problema, sino la escasez de información y concienciación? 


\section{7.-Análisis de resultados.}

Respecto al análisis de resultados, a modo introductorio, se ha de mencionar que se procede a la interpretación de los datos, respuestas y resultados obtenidos tanto en el análisis descriptivo como en el análisis correlacional de Rho de Spearman, así como del cuestionario de Escala Likert como de las entrevistas.

En primer lugar, se detallan unas pequeñas interpretaciones de las respuestas recibidas por parte de los sujetos entrevistados. La inmensa mayoría de respuestas a la entrevista individual realizada coincide en que, actualmente hay una falta de información y conocimiento acerca de todo lo referente a las "Necesidades Educativas Especiales", incluso desconociendo por completo las siglas "N.E.E", de ahí las peticiones constantes de reuniones y charlas informativas para adquirir mayor contenido e información sobre la temática. Además, la mayoría coinciden en que los propios docentes no están preparados para tratar a los discentes que presentan cualquier tipo de necesidad especial, retrasando de este modo, su proceso de enseñanza y aprendizaje dentro del aula ordinaria, así como, destacan la importancia de concienciar a los más pequeños de la igualdad de oportunidades, capacidades y posibilidades que tienen todos los niños, a pesar de presentar cualquier tipo de Necesidad Educativa Especial o no.

Por otra parte, haciendo alusión al análisis descriptivo propiamente dicho, de entre los 25 ítems del cuestionario de escala Likert, se han seleccionado los histogramas más significativos y los que muestran con mayor claridad y relevancia los datos que se pretendía obtener para la contrastación de hipótesis y consecución de objetivos.

\section{Gráfico 1.-A4.}

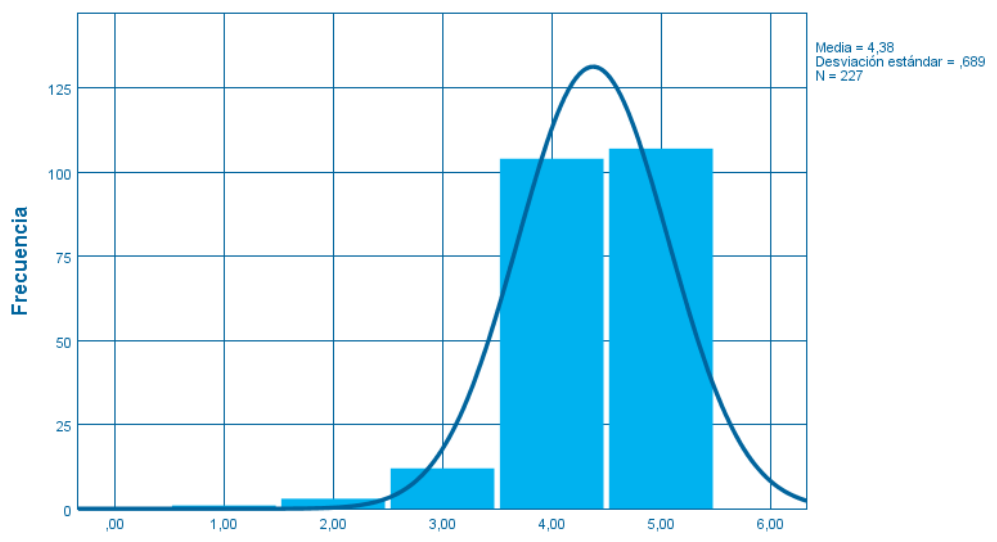

A4. Las habilidades sociales de los niños/as se ven claramente afectadas por su entorno más cercano.

Fuente: elaboración propia. 
Histograma 1. A4. Las habilidades sociales de los niños/as se ven claramente afectadas por su entorno más cercano. Recuperado de IBM SPSS Statistics 25. Siendo la media de una puntuación de 4,38, se verifica que los participantes están de acuerdo con este ítem y con que, el contexto inmediato en el que se desenvuelven los niños afecta de manera directa al desarrollo de sus habilidades sociales, puesto que la variedad de respuestas ha sido pequeña, como muestra la puntuación de la desviación estándar ,689.

\section{Gráfico 2.-B7.}

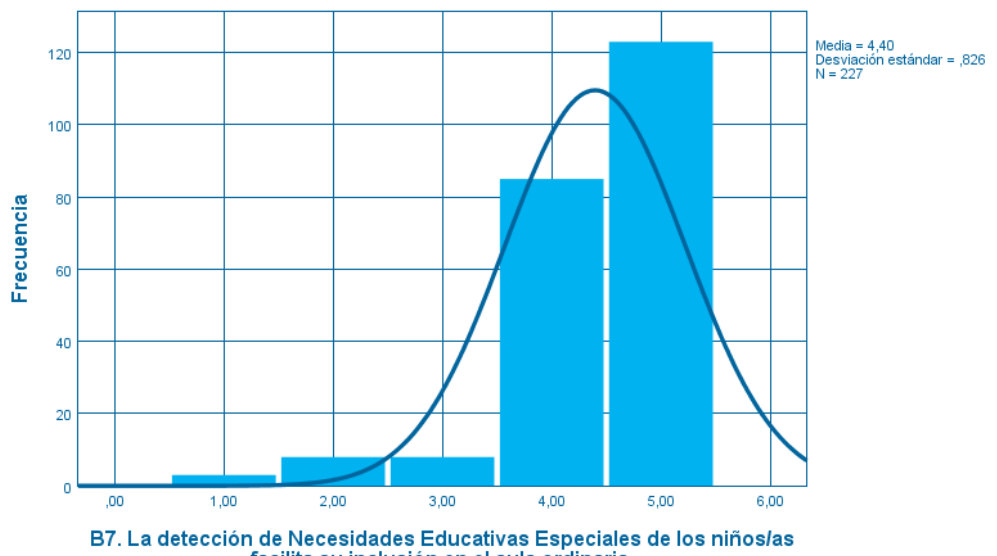

facilita su inclusión en el aula ordinaria.

Fuente: elaboración propia.

Histograma 2. B7. La detección de Necesidades Educativas Especiales de los niños/as facilita su inclusión en el aula ordinaria. Recuperado de IBM SPSS Statistics 25. Los sujetos participantes de la investigación vuelven a estar de acuerdo con este ítem, demostrando la desviación estándar, poca variación dentro del grado de conformidad, por lo que se hace necesario el diagnóstico de Necesidades Educativas Especiales para que se alcance la inclusión educativa dentro del aula ordinaria. 


\section{Gráfico 3.-D28.}

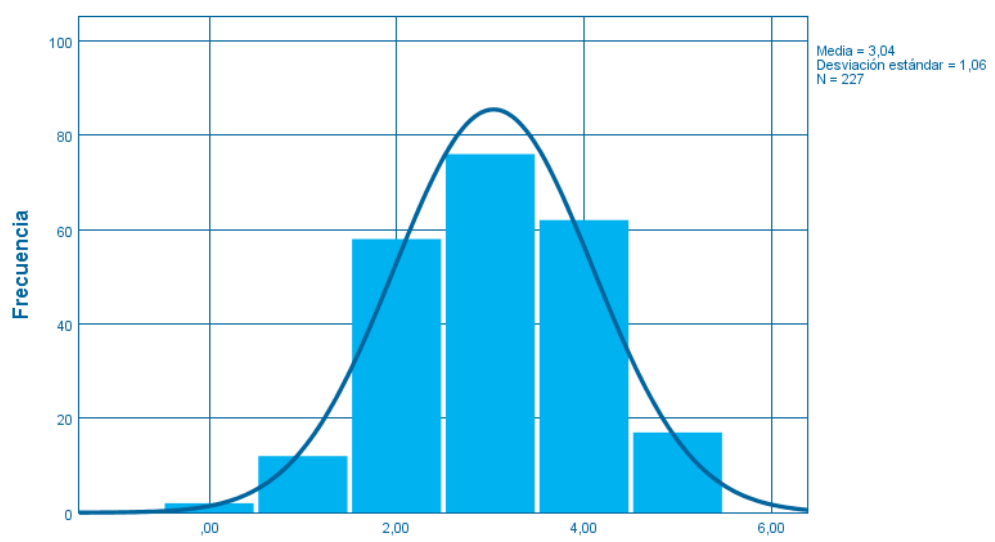

D18. Tengo una amplia información acerca de si mi hijola asiste a un colegio inclusivo.

Fuente: elaboración propia.

Histograma 6. D18. Tengo una amplia información acerca de si mi hijo/a asiste a un colegio inclusivo. Recuperado de IBM SPSS Statistics 25. En este sentido, la población demuestra indiferencia ante esta cuestión que se les plantea, aunque hay una desviación estándar de un 1,06 que señala la variedad de respuestas, se verifica y contrasta que realmente no se tiene la información que se debería sobre si existe una real inclusión educativa en la actualidad.

Por último, en cuanto al análisis correlacional, en la siguiente tabla se detallan todas las correlaciones de cada uno de los ítems del cuestionario. Las correlaciones más representativas son las que están subrayadas y de entre ellos, se ha hecho una selección de las más significativas, eligiendo concretamente tres, las más destacadas de la tabla, son B7. $\Leftrightarrow$ D16. $400^{* *}, B 8 . \Leftrightarrow$ C13. $465^{* *} y$ C15. $\Leftrightarrow$ E23. $465^{\star *}$.

Por consecuencia, de las correlaciones destacadas y escogidas se va a realizar una breve interpretación y análisis de relación entre las cuestiones, para ver el grado de relación y conexión que existe entre ambos ítems.

Existe una correlación positiva entre inclusión educativa y comportamiento del profesorado, de modo que la conducta y labor docente favorece de manera directa la inclusión de los niños con N.E.E en el aula ordinaria. Por otro lado, también existe una correlación positiva entre identificación de N.E.E y la forma de actuar ante ellas, de tal forma que, cuanta más información y habilidades tenemos para saber identificar y diagnosticar cualquier problemática 0 necesidad presentes en los educandos, mejor será nuestra capacidad para actuar y tratar a los mismos, Y, por último, se presenta una correlación positiva entre pautas de actuación y aceptación con obtención de información, demostrando que para actuar y proceder ante la 
tipología de Necesidades Educativas Especiales, es preciso poseer la información y conocimiento necesarios.

Tabla 2. Análisis correlacional Rho de Spearman.

\section{Correlaciones Rho de Spearman}

\begin{tabular}{|c|}
\hline A1. $\Leftrightarrow$ A4. ,299** \\
\hline A2. $\Leftrightarrow$ B9. , 364** \\
\hline A3. $\Leftrightarrow$ B7. ,300** \\
\hline A4. $\Leftrightarrow$ A5. ,526** \\
\hline A5. $\Leftrightarrow$ A5. ,526** \\
\hline B6. $\Leftrightarrow$ C11. $388^{* *}$ \\
\hline B7. $\Leftrightarrow$ D16. $400^{* *}$ \\
\hline B8. $\Leftrightarrow$ C13. $465^{* *}$ \\
\hline B9. $\Leftrightarrow$ A2. ,364** \\
\hline B10. $\Leftrightarrow$ A2. ,228** \\
\hline C11. $\Leftrightarrow$ C13. ,609** \\
\hline C12. $\Leftrightarrow$ E25. ,390** \\
\hline C13. $\Leftrightarrow$ C11. ,609** \\
\hline C14. $\Leftrightarrow$ C15. ,456** \\
\hline C15. $\Leftrightarrow$ E23. $465^{* *}$ \\
\hline D16. $\Leftrightarrow$ E21. ,487** \\
\hline D17. $\Leftrightarrow$ C14. ,376** \\
\hline D18. $\Leftrightarrow$ D19. ,442** \\
\hline D19. $\Leftrightarrow$ D18. ,442** \\
\hline D20. $\Leftrightarrow$ E21. ,351** \\
\hline E21. $\Leftrightarrow$ E22. ,664** \\
\hline E22. $\Leftrightarrow$ E23. $717^{* *}$ \\
\hline E23. $\Leftrightarrow$ E21. ,633** \\
\hline E24. $\Leftrightarrow$ E22. ,541** \\
\hline E25. $\Leftrightarrow$ C12. ,390** \\
\hline
\end{tabular}

Fuente: elaboración propia.

\section{4.-Conclusión.}

A modo de conclusión, al inicio de la investigación se planteó el objetivo general "Identificar las Necesidades Educativas Especiales que presentan los niños y su influencia en la inclusión de los mismos", en torno al cual iba a tratar todo el desarrollo del trabajo".

Por otro lado, se ha verificado y contrastado la hipótesis $0(\mathrm{HO})$ de que la detección de Necesidades Educativas Especiales ayuda a la manera de incluir a los niños en el aula ordinaria, promoviendo la aparición de una verdadera inclusión educativa en el aula ordinaria. 
Así como, la consecución de los objetivos específicos planteados de manera inicial, puesto que se ha comprobado que el ambiente y contexto inmediato en el que se desarrolla un niño afecta de manera directa al desarrollo de sus habilidades sociales, favoreciendo o de lo contrario, perjudicando, las relaciones sociales que mantengan los discentes a lo largo de su vida.

Además, se destaca la importancia de distinguir la diversidad de Necesidades Educativas Especiales y, por consecuencia, las diferentes formas de actuar ante ellas. Y, el análisis e indagación para averiguar y descubrir si realmente existe o no una verdadera inclusión educativa dentro del aula ordinaria.

Igualmente, se ha verificado que la población, que en representación de la sociedad ha participado en esta investigación, demanda una mayor información, conocimiento y asesoría acerca de la temática tratada en cuestión, para de esta forma poder tomar medidas y desarrollar pautas de actuación ante las diversas situaciones que les pueda surgir con los niños y niñas, en un determinado momento, presenten cualquier clase de Necesidades Educativas Especiales.

Finalmente, tanto con el análisis descriptivo como con el correlacional se han analizado, asociado y comparado diferentes grupos de datos, comprobando el grado de relación e influencia entre los distintos ítems que conforman el cuestionario de escala Likert y, gracias a estos, las entrevistas individuales. Ambas técnicas han dado la oportunidad de avanzar en esta importante temática de interés y ha ayudado a solventar posibles dudas que podía haber y puede surgir en un futuro, ayudando así a la aportación de nueva información y de una nueva investigación que puede apoyar indagaciones, búsquedas y estudios venideros.

\section{5.-Referencias.}

Alonso, M. A. V., Aguilella, A. R. (2008). Valoración de la inclusión educativa desde diferentes perspectivas. Siglo Cero: Revista Española sobre Discapacidad Intelectual, 39(228), 5-25.

Chiner, E. S. (2011). Las Percepciones y Actitudes del Profesorado hacia la Inclusión Del Alumno con Necesidades Educativas Especiales como Indicadores del Uso de Prácticas Educativas Inclusivas en el Aula. (Tesis de Doctorado). Universidad de Alicante. Departamento de Psicología de la Salud. Alicante.

Comisión de Expertos de Educación Especial (2004). Informe de la Comisión de Expertos: nueva perspectiva y visión de la educación especial. Ministerio de Educación. Recuperado de http://especial.mineduc.cl/wpcontent/uploads/sites/31/2016/08/201304151157200.Doc_Nueva_perspecti va_vision_Ed_Especial.pdf

Instrucciones de 8 de Marzo de 2017, de la Dirección General de Participación y Equidad, por las que se establece el protocolo de detección, identificación del alumnado con Necesidades Específicas de Apoyo Educativo y Organización de la respuesta educativa. BOJA. Recuperado de http://www.juntadeandalucia.es/educacion/portals/web/ced/normativa//normativas/detalle/instrucciones-de-8-de-marzo-de-2017-de-la-direccion- 
general-de-participacion-y-equidad-por-las-que-se-actualiza-el1xr2aw1d841lt

Rodríguez, D. E. C., \& Ortiz, J. J. B. (2017). Gestión del conocimiento para la adaptación curricular de estudiantes con necesidades educativas especiales. Gestion, competitividad e innovación, 5(1). Recuperado de https://scholar.google.es/scholar?hl=es\&as_sdt=0\%2C5\&q=Gesti\%C3\%B3 $\mathrm{n}+$ del+conocimiento+para+la+adaptaci\%C3\%B3n+curricular+de+estudiant es+con+necesidades+educativas+especiales\&btnG=

Unesco (1994). Declaración de Salamanca y Marco de acción para las Necesidades Educativas Especiales. Conferencia Mundial sobre Necesidades Educativas Especiales: Acceso y Calidad. Salamanca: Ministerio de Educación y Ciencia. Recuperado de: https://unesdoc.unesco.org/ark:/48223/pf0000098427_spa

Unesco (2008). Conferencia Internacional de Educación. La educación inclusiva: El camino hacia el futuro. París. Recuperado de http://www.ibe.unesco.org/fileadmin/user_upload/Policy_Dialogue/48th_ICE /General_Presentation-48ClE-4_Spanish_.pdf 